\title{
Sarcoidosis and coeliac disease: do not forget the association!
}

\author{
Cristina D'Ercole • Angelo Zullo • \\ Maria Consiglia Bragazzi • \\ Salvatore Maria Antonio Campo
}

Received: 5 January 2011/Accepted: 6 March 2011/Published online: 23 March 2011

(C) SIMI 2011

\begin{abstract}
A 70-year-old woman, with sarcoidosis localized in the lungs and cervical lymph nodes since 2008, presented to our open access Endoscopic Unit in May 2010 for colonoscopy due to iron deficiency anaemia (haemoglobin $=10.9 \mathrm{~g} / \mathrm{dL}, \mathrm{MCV}=72 \mathrm{fl}$, iron $=25 \mathrm{mg} / \mathrm{dL}$ ). The patient did not present any abdominal symptoms, and denied rectal bleeding. At colonoscopy, a $5 \mathrm{~mm}$ in diameter sessile polyp localized in the sigmoid colon was removed with the snare. Since no evident causes for anaemia were discovered, we suggested an upper endoscopy in the same endoscopic session to rule out other potential causes of blood loss. Surprisingly, reduced duodenal folds in the duodenum with 'scalloping' signs were found. Histological assessment of duodenal biopsies showed a total villous atrophy indicative of coeliac disease (CD), which was confirmed by positivity of both antiendomisium and anti-transglutaminase antibodies in the serum. The patient started a gluten-free diet obtaining a complete regression of anaemia in a few months.
\end{abstract}

Sarcoidosis is a chronic idiopathic granulomatous disease as a consequence of altered immune response to unidentified antigens. Sarcoidosis mainly involves the lungs, even though other organs may be involved. Moreover, sarcoidosis has been associated with different autoimmune disorders, such as primary biliary cirrhosis, Crohn's disease, and Sjogren's syndrome [1].

An association between sarcoidosis and CD has been also described [2]. Although the mechanisms involved remain unclear, both diseases seem to share some immunological and genetic disorders. Indeed, the susceptibility of

C. D’Ercole · A. Zullo · M. C. Bragazzi · S. M. A. Campo ( $\square)$ Gastroenterology Unit, Ospedale Nuovo Regina Margherita, Rome, Italy

e-mail: salvatorecampo@yahoo.com developing both sarcoidosis and $\mathrm{CD}$ has been linked to the class II haplotype HLA-DR3, DQ2 and B8 [3]. It is also possible that, by increasing the expression of class II HLA molecules, the diseases predispose to each other [3]. Thus the presence of $\mathrm{CD}$ may increase the risk of sarcoidosis development and vice versa $[4,5]$. In detail, $C D$ has been associated with an increased risk of sarcoidosis ( $\mathrm{HR}=$ 4.03 ; $95 \%$ CI $=2.32-7.00$ ). Similarly, a prior sarcoidosis diagnosis is associated with an increased risk of $\mathrm{CD}$ with an OR of 3.58 (95\% CI = 1.98-6.45). Nevertheless, sarcoidosis often occurs in older subjects, while CD is more frequently observed in young people. Therefore, the association may not be observed by the physicians, especially when considering that $\mathrm{CD}$ in adults may present exclusively with an iron deficiency anaemia without any gastrointestinal symptoms, as was the case in our patient.

In conclusion, CD should be promptly sought in all patients with sarcoidosis and a unexplained iron deficiency anaemia, before performing more invasive diagnostic tests.

Conflict of interest None.

\section{References}

1. James DG, Sharma OP (1985) Overlap syndromes with sarcoidosis. Postgrad Med J 61:769-771

2. Rutherford RM, Brutsche MH, Kearns M, Bourke M, Stevens F, Gilmartin JJ (2004) Prevalence of coeliac disease in patients with sarcoidosis. Eur J Gastroenterol Hepatol 16:911-915

3. Reggoug S, Benelbarhdadi I, Essamri W, Ajana FZ, Afifi R, Benazzouz M, Essaid A (2009) Celiac disease associated with sarcoidosis. Gastroenterol Clin Biol 33:430-432

4. Ludvigsson JF, Wahlstrom J, Grunewald J, Ekbom A, Montgomery SM (2007) Coeliac disease and risk of sarcoidosis. Sarcoidosis Vasc Diffuse Lung Dis 24:121-126

5. Hwang E, McBride R, Neugut AI, Green PH (2008) Sarcoidosis in patients with celiac disease. Dig Dis Sci 53:977-981 